\title{
Intrinsic and Extrinsic Ferromagnetism in Co-Doped Indium Tin Oxide Revealed Using X-Ray Magnetic Circular Dichroism
}

\author{
A. M. H. R. Hakimi, ${ }^{1}$ F. Schoofs, ${ }^{1}$ M. G. Blamire, ${ }^{1}$ S. Langridge, ${ }^{2}$ and S. S. Dhesi ${ }^{3}$ \\ ${ }^{1}$ Department of Materials Science and Metallurgy, University of Cambridge, 27 Charles Babbage Road, Cambridge CB3 OFS, UK \\ ${ }^{2}$ Science and Technology Facilities Council, Rutherford Appleton Laboratory, Harwell Science and Innovation Campus, \\ Didcot OX11 0QX, UK \\ ${ }^{3}$ Diamond Light Source, Chilton, Didcot, Oxfordshire OX11 ODE, UK
}

Correspondence should be addressed to S. S. Dhesi; dhesi@diamond.ac.uk

Received 23 February 2017; Accepted 9 March 2017; Published 13 April 2017

Academic Editor: Ward Beyermann

Copyright (C) 2017 A. M. H. R. Hakimi et al. This is an open access article distributed under the Creative Commons Attribution License, which permits unrestricted use, distribution, and reproduction in any medium, provided the original work is properly cited.

\begin{abstract}
The effects of high-temperature annealing on ferromagnetic Co-doped Indium Tin Oxide (ITO) thin films have been investigated using X-ray diffraction (XRD), magnetometry, and X-Ray Magnetic Circular Dichroism (XMCD). Following annealing, the magnetometry results indicate the formation of Co clusters with a significant increase in the saturation magnetization of the thin films arising from defects introduced during cluster formation. However, sum rule analysis of the element-specific XMCD results shows that the magnetic moment at the Co sites is reduced after annealing. The effects of annealing demonstrate that the ferromagnetism observed in the as-deposited Co-doped ITO films arises from intrinsic defects and cannot be related to the segregation of metallic Co clusters.
\end{abstract}

\section{Introduction}

Dilute magnetic oxides, formed by randomly substituting magnetic transition-metal (TM) ions into a host oxide, have attracted significant interest in recent years due to their potential application in spintronic devices $[1,2]$. On the other hand room temperature ferromagnetism remains controversial in these materials because the classification of intrinsic or extrinsic ferromagnetism has proved particularly difficult [35]. Furthermore, ferromagnetism has been reported in oxides without TM ion doping $[6,7]$ calling into question the precise role of the TM impurities in establishing the ferromagnetic ordering.

A case in point is TM-doped Indium Tin Oxide (ITO) which exhibits room temperature ferromagnetism and has sparked considerable interest in its use as a transparent spin valve [8-12]. However, for optically transparent Co-doped ITO the Co ions have been observed to remain paramagnetic at low temperature $(T=2 \mathrm{~K})$ and hence do not contribute to the long range ferromagnetic ordering [13]. Instead, calculations indicate that ferromagnetism is related to defect concentration rather than TM ion doping [14]. In particular, oxygen vacancies may play an important role in the emergence of ferromagnetism in these materials $[15,16]$. Baqiah et al. recently showed that the magnetic behavior of Codoped ITO films made by a sol-gel process was sensitive to both the Co doping concentration and the density of oxygen vacancies as well as the estimated ratio of oxygen defects to Co ions [17]. Unfortunately, direct evidence for intrinsic ferromagnetism in this material remains elusive, but nowadays powerful element and site-specific probes such as X-Ray Magnetic Circular Dichroism (XMCD) and X-Ray Absorption Spectroscopy (XAS) are proving invaluable.

Here, we focus on ferromagnetic ordering in as-deposited and ultrahigh vacuum (UHV) annealed Co-doped ITO films using magnetometry together with XAS and XMCD. The annealing process is found to increase the saturation magnetization but decrease the magnetic moment per Co ion. This suggests that the ferromagnetism in the annealed films has a strong contribution from metallic Co clusters in contrast to the ferromagnetic response in the as-deposited 

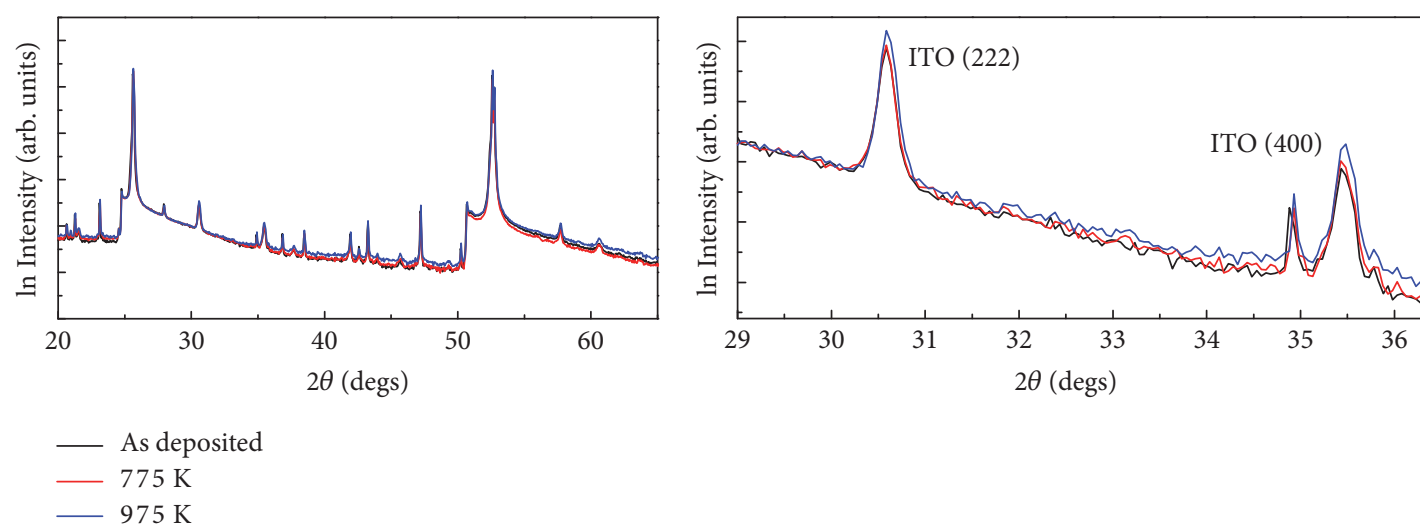

(a)

(b)

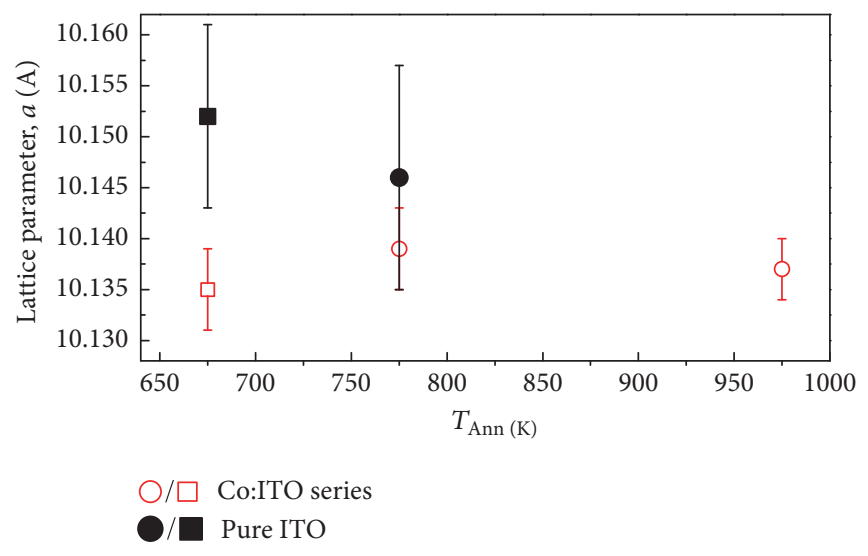

(c)

FIGURE 1: (a) $\theta$-2 $\theta$ XRD diffractograms of the as-deposited (solid black line) and annealed films (red and blue solid lines); (b) an expanded view of the same $\theta-2 \theta$ XRD diffractograms shown in (a) but focus is now put on the ITO (222) and ITO (400) peaks demonstrating the shift in the peak positions as the annealing temperature is increased to $975 \mathrm{~K}$; (c) out-of-plane lattice parameter for as-deposited (squares) and annealed (circles) pure ITO (black) and Co-doped ITO (red) thin films.

films which we believe to be intrinsic and free from metallic clustering. In addition to this, magnetometry measurements reveal a ferromagnetic blocking temperature for the annealed films giving further evidence for metallic clustering.

\section{Experimental Details}

Co-doped (5.4 at.\%) ITO thin films were codeposited using DC magnetron sputtering in a UHV system with a base pressure $<1 \times 10^{-6} \mathrm{~Pa}$. The $250 \mathrm{~nm}$ thick films were grown at $675 \mathrm{~K}$ on $r$-plane sapphire substrates. A highly oxygendeficient environment was used to create significant oxygen deficiency in the films, most likely in the form of oxygen vacancies. Films were sputtered in $1.2 \pm 0.01 \mathrm{~Pa}$ of an Ar$\mathrm{O}_{2}(95: 5)$ mix $\left(0.06 \mathrm{~Pa} \mathrm{O}_{2}\right)$. UHV annealing was performed for 2 hours in the deposition chamber at $975 \mathrm{~K}$. Magnetic characterization was carried out with a superconducting quantum interference device (SQUID) over a temperature range $2-300 \mathrm{~K}$ using magnetic fields of up to $0.5 \mathrm{~T}$. XAS and XMCD measurements were performed over the Co $L_{2,3}$ edges ( $2 p \rightarrow 3 d$ dipole transitions) on beamline I06, Diamond Light Source, using the UHV compatible superconducting high-field magnet which provides fields of up to $6 \mathrm{~T}$ and operates in a temperature range of $2-370 \mathrm{~K}$. The sample was mounted on a custom-designed pure $\mathrm{Cu}$ block using silver paste for good electrical contact. All SQUID magnetic characterization was performed before the XAS and XMCD measurements. The samples were carefully handled, that is, using nonmagnetic tweezers, to minimize the possibility of contamination leading to a false ferromagnetic effect.

\section{Results and Discussion}

3.1. Structural Properties. X-ray diffraction was performed on a Bruker D8 diffractometer in Bragg-Brentano geometry with $\mathrm{Cu} \mathrm{K} \alpha$ radiation and a position sensitive detector. Xray diffraction of the as-deposited and annealed films could be indexed assuming the cubic bixbyite structure of the pure ITO [18]. Lattice parameters were calculated based on profile-fitted cubic ITO reflections (211), (222), (400), (440), and (622), using the sapphire (001) reflections as an internal standard. The diffraction peaks were sharp, suggesting a high degree of crystallinity, with no detectable peaks corresponding to secondary Co oxides. Figure 1 shows $\theta-2 \theta$ 
XRD scans of the as-deposited and that of the sample annealed at $975 \mathrm{~K}$. To verify the absence of Co clusters further in our as-deposited samples, the integration time per point was raised significantly to 100 seconds. This still yielded spectra suggesting the absence of any metallic cluster formation. The same procedure was carried out on the annealed samples ending in the same outcome: within the detection limits of the instrument, no clusters could be detected even after hightemperature UHV annealing. Figure 1(b) shows an expanded view of the predominant ITO (222) peak and that of the ITO (400) peak for the as-deposited and two other films annealed at different temperatures.

There is no obvious significant shift in the ITO (222) peak position as the annealing temperature is changed. The only obvious change is the increased intensity of the ITO (222) peak at the highest annealing temperature ( $975 \mathrm{~K})$, perhaps suggesting a marked increase in crystalline quality. However, there is a clear shift in the peak position of the ITO (400) peak towards higher values for $2 \theta$ as the annealing temperature is increased and would suggest a decrease in the out-of-plane lattice parameter, $a$. This is consistent with our own findings for pure ITO thin films which have been grown and annealed at different temperatures [19].

Figure 1(c) shows the shifts in $a$ as a function of the annealing temperature for pure and Co-doped ITO thin films. It is clear that samples which have been doped with Co (red data points) have a lower $a$. This can be explained by the substitution of $\mathrm{Co}^{2+}$, which has a smaller ionic radius, for $\mathrm{In}^{3+}$ within the ITO matrix. We have shown in a previous study that $a$ will continue to decrease with increasing levels of Co doping [13]. Annealing the samples at $775 \mathrm{~K}$ resulted in a small increase in $a$ and may correspond to the migration of $\mathrm{Sn}$ ions in the ITO matrix and the out-diffusion of $\mathrm{Co}^{2+}$ ions from $\mathrm{In}^{3+}$ lattice sites, resulting in the formation of interstitial metallic Co clusters. Increasing the annealing temperature further may compound this effect and also result in increased out-diffusion of oxygen from interstitial sites. This could result in the relaxation of the lattice and explain the apparent drop in $a$ at higher $975 \mathrm{~K}$. We note that these changes are very small and are within the specified experimental error. Therefore, from XRD alone we can only speculate such mechanisms upon annealing. Although there is no evidence for Co clustering from the XRD data, evidence that we present later suggests otherwise and again demonstrates the need for more powerful techniques to probe the true nature of magnetism in these materials.

3.2. Electrical Properties. The films were metallic with a room temperature carrier concentration (determined via Hall measurements using the van der Pauw configuration) of $8.5 \times 10^{20} \mathrm{~cm}^{-3}$ which increased to $9.5 \times 10^{20} \mathrm{~cm}^{-3}$ after annealing. This implies that the annealing process introduced additional oxygen vacancies into the film along with improved substitution of $\mathrm{Sn}$ ions for In ions. Both the improved incorporation of Sn ions into the matrix and the creation of oxygen vacancies provide extra carriers for conduction.

3.3. Magnetic Properties. In-plane magnetization (M) versus field $\left(\mu_{0} H\right)$ loops measured at room temperature for the as-deposited and annealed films are shown in Figure 2(a). The diamagnetic background of the substrate and sample holder has been subtracted from all the magnetization data shown here. The maximum saturation magnetization, $M_{S}$, for the as-deposited film is $1.02 \mathrm{emu} \mathrm{cm}^{-3}$ which increases to $2.55 \mathrm{emu} \mathrm{cm}^{-3}$ after annealing. In addition, the coercive field increases from $\sim 8 \mathrm{mT}$ to $\sim 34 \mathrm{mT}$ after annealing.

Figure 2(b) shows the temperature dependence of the zero-field cooled (ZFC) and field-cooled (FC) magnetization measured in a field of $10 \mathrm{mT}$ for the annealed Co-doped ITO film. There is a clear separation between the ZFC and FC curves at $\sim 272 \mathrm{~K}$ which represents the superparamagnetic blocking temperature, $T_{B}$, and indicates the presence of magnetic nanoclusters. The value of $T_{B}$ can be used to estimate the cluster size using the relationship $T_{B}=K_{\mathrm{Co}} V / k_{B} \ln \left(\tau_{m} / \tau_{0}\right)$, where $K_{\mathrm{Co}}$ is the magnetic anisotropy energy density for Co $\left(4.9 \times 10^{5} \mathrm{~J} \mathrm{~m}^{-3}\right), V$ is the volume of the nanoparticle, $k_{B}$ is the Boltzmann constant, $\tau_{m}$ is the experimental measurement time (taken to be $10 \mathrm{~s}$ ), and $\tau_{0}$ is the attempt period ( $1 \times$ $\left.10^{-10} \mathrm{~s}\right)$. Assuming spherically shaped clusters, the particle radius is estimated to be $3.8 \pm 0.1 \mathrm{~nm}$. The $\mathrm{ZFC}-\mathrm{FC}$ magnetization curve for the as-deposited sample showed no evidence of a superparamagnetic $T_{B}$ [13]. This clearly indicates that, aside from an increase in oxygen vacancy concentration as evidenced from the increase in carrier density, Co clusters are formed due to preferential reduction of $\mathrm{Co}^{2+}$ ions in the reducing environment.

Further evidence to support the role that oxygen vacancies have on these films is demonstrated in Figure 3 which shows a series of room temperature magnetization loops for 5.4 at.\% Co-doped ITO thin films grown under varying oxygen partial pressures. The figure clearly shows the reduction in the strength of the magnetic response as the oxygen partial pressure during growth increases. The inset also shows a clear correlation between magnetization and the carrier concentration: increasing carrier concentration results in an increase in the magnetic response (as was also seen by Stankiewicz et al. for their Co-doped ITO films) [8]. This gives clear evidence that oxygen vacancies play a crucial role in mediating magnetism in these films. Coey et al. proposed a bound magnetic polaron (BMP) mechanism involving oxygen vacancies to explain the ferromagnetic behavior in these TM-doped oxide films [20]. These vacancies are capable of forming so-called $F$-centers with trapped electrons. The overlapping of $F$-center electron orbitals with the $d$ orbitals of neighboring TM dopants to form TM-oxygen vacancy-TM groups is key to the observed ferromagnetism.

3.4. XAS and XMCD Measurements. XAS and XMCD measurements were performed over the Co $L_{2,3}$ edge at $2 \mathrm{~K}$ in an applied field of $4 \mathrm{~T}$. The sample surface was oriented at $45^{\circ}$ to the incident beam and the magnetic field was applied parallel to the direction of the incident X-ray beam. XAS spectra were recorded in total-electron yield (TEY) and fluorescence yield (FY) mode in order to measure the surface and bulk components, respectively. Figure 4 shows the TEY XAS spectra for a sample magnetization parallel $\left(\rho_{+}\right)$and antiparallel $\left(\rho_{-}\right)$to the photon helicity vector for a pure Co foil, the as-deposited film, and the annealed film. The XAS 


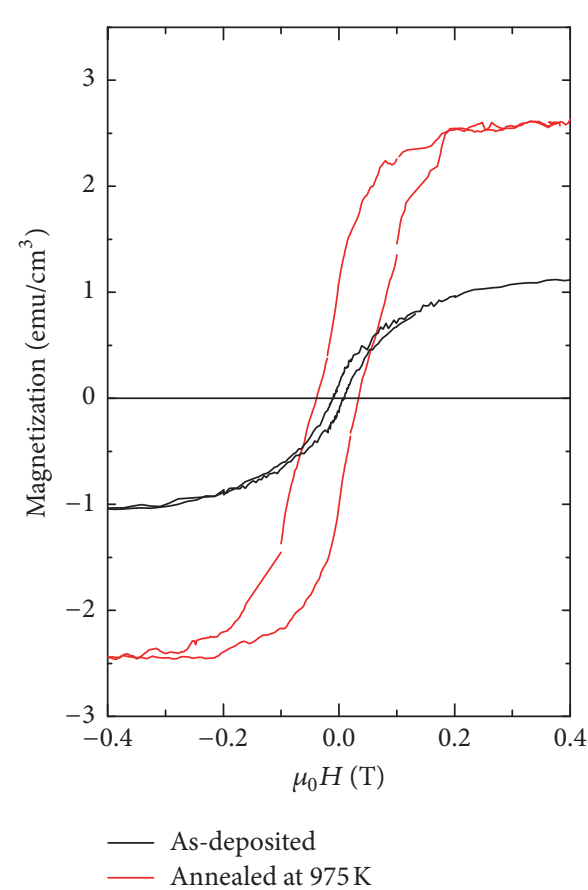

(a)

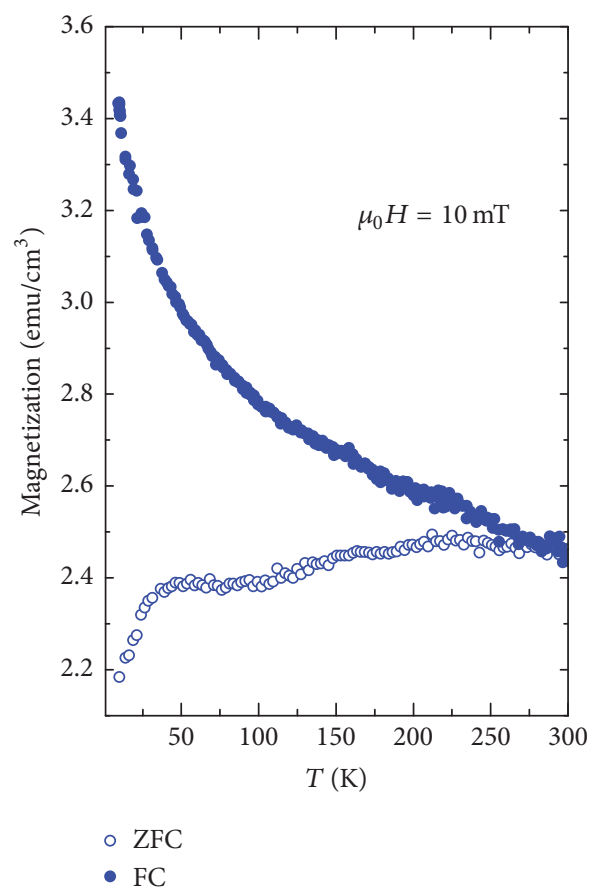

(b)

FIgURE 2: (a) Room temperature hysteresis loops for an as-deposited (black line) and annealed (red line) Co-doped ITO. (b) Zero-field cooled (open blue circles) and field-cooled (solid blue circles) magnetization versus temperature curves for the annealed Co-doped ITO film recorded in an applied magnetic field of $10 \mathrm{mT}$.

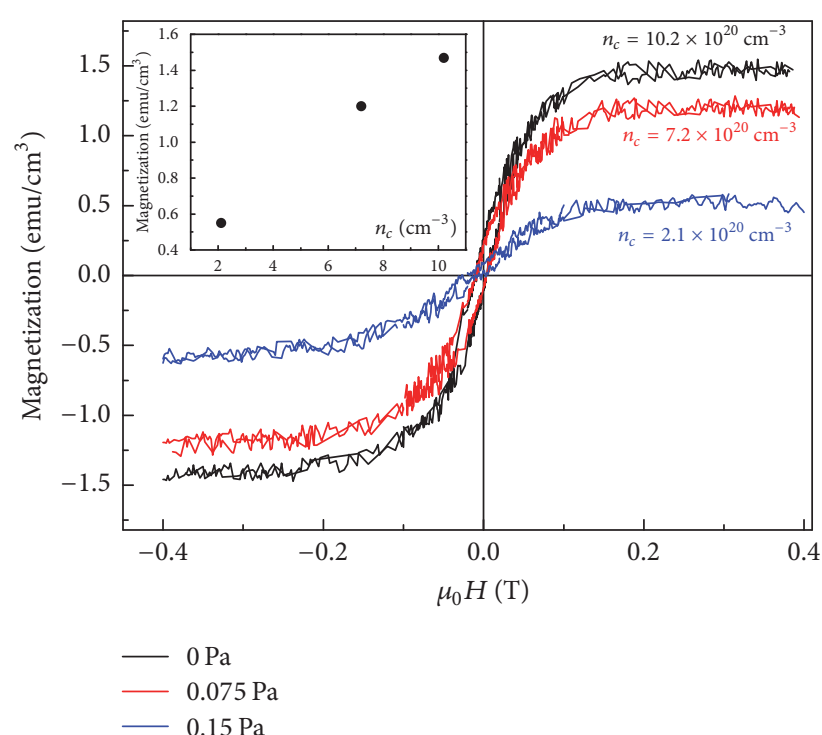

FIGURE 3: Room temperature magnetization versus applied field loops for 5.4 at.\% Co-doped ITO thin films grown under varying oxygen partial pressures. Corresponding carrier concentrations are also given. Inset: room temperature magnetization as a function of carrier concentration for the same films.

and XMCD $\left(\rho_{-}-\rho_{+}\right)$from the Co foil shown in Figures 4(a) and 4(d), respectively, are representative of metallic Co [21]. On the other hand, the pronounced XAS multiplet structure at the Co $L_{2,3}$ edges for the as-deposited film (Figure 4(b)) arises from Coulomb and exchange interactions between $2 p$ core hole and $3 d$ valence states and is typical for a $\mathrm{Co}^{2+}$ ion. After annealing the XAS multiplet features at $L_{3}$ peak shown in Figure 4(c) are less pronounced, indicating an increased fraction of metallic Co. Figures 4(e) and 4(f) also show the corresponding XMCD spectra. The XMCD spectrum for the annealed sample (Figure 4(f)) exhibits features which are a mixture of the metallic and $\mathrm{Co}^{2+}$ spectra $[13,22]$ suggesting the presence of metallic and ionic $\mathrm{Co}^{2+}$ near the surface region after annealing.

The presence of metallic Co in the annealed Co-doped ITO sample was also confirmed by XAS and XMCD spectra measured in FY mode and is shown in Figure 5. Comparison of the XAS and XMCD spectra for the as-deposited and annealed films shows that the multiplet structure has been largely removed after annealing. The lineshape of the spectra is closer to that of metallic Co and suggests that the bulk of the film contains metallic Co clusters. This is consistent with the superparamagnetic blocking temperature measured in $M$ $T(\mathrm{~K})$ data shown in Figure 2(b). The arrows in Figure 5(a) indicate the emergence of two new features in the XAS spectrum which only appear after the annealing treatment. These new features may indicate hybridization of Co states to oxygen or In after the annealing process.

To probe the origins of the magnetism in the annealed films further, XAS and XMCD measurements were performed at $4 \mathrm{~K}$ and at remanence. The multiplet fine structure was still present in both TEY and FY XAS spectra, but no 


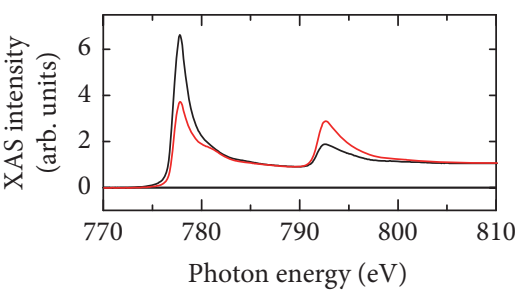

(a)

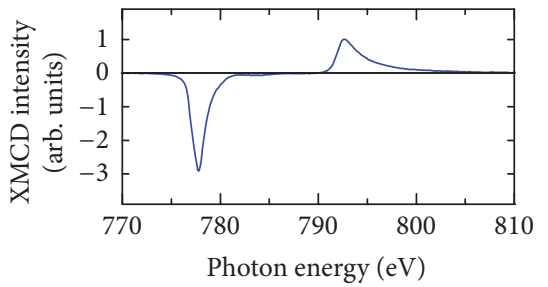

(d)

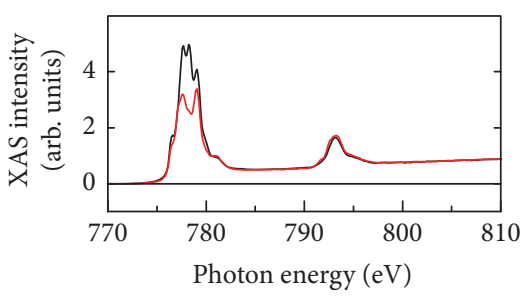

(b)

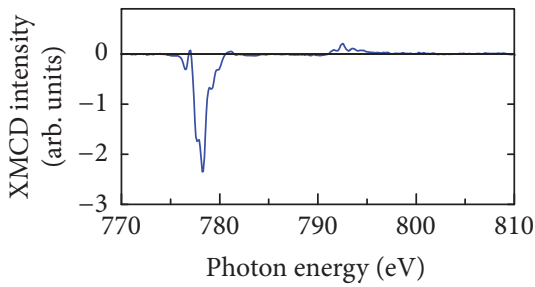

(e)

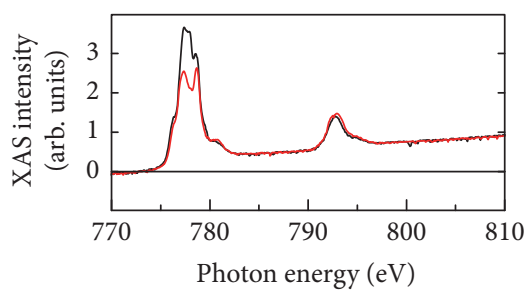

(c)

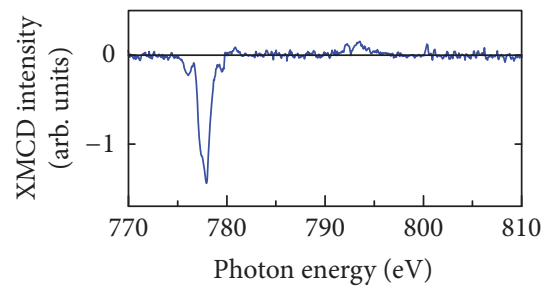

(f)

FIGURE 4: TEY XAS spectra taken with $\rho_{+}$(black line) and $\rho_{-}$(red line) X-rays for (a) Co metal, (b) as-deposited Co-doped ITO, and (c) annealed Co-doped ITO at $2 \mathrm{~K}$ in an applied field of 4 T. Corresponding XMCD spectra for (d) Co metal, (e) as-deposited Co-doped ITO, and (f) annealed Co-doped ITO.

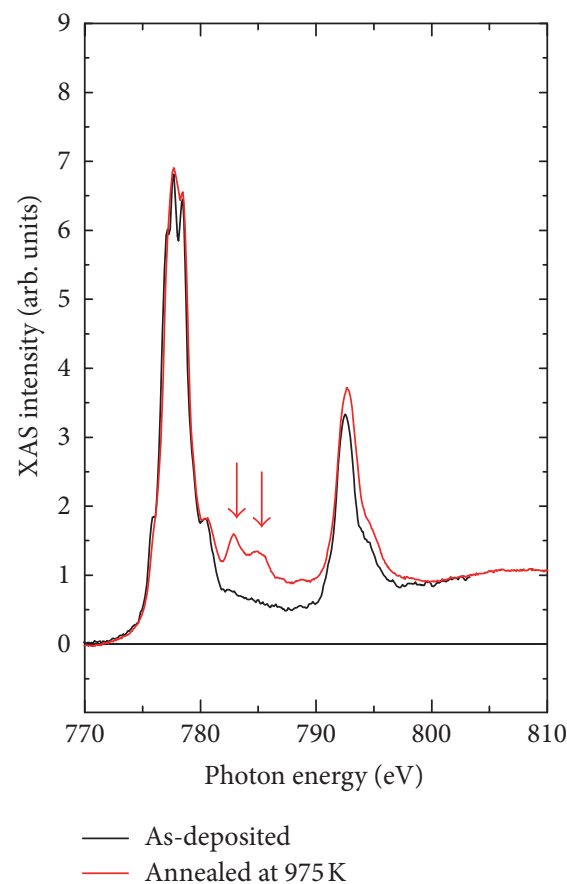

(a)

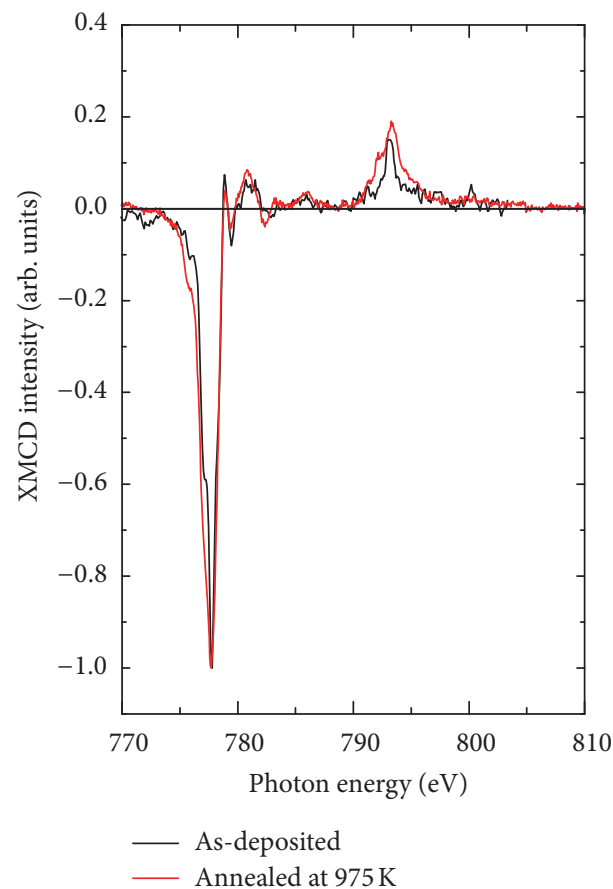

(b)

FIGURE 5: (a) Isotropic FY XAS spectra for the as-deposited (black line) and annealed (red line) Co-doped ITO film. Results recorded at $2 \mathrm{~K}$ in an applied magnetic field of $4 \mathrm{~T}$. The arrows indicate spectral features that arise after annealing. (b) XMCD spectra for the as-deposited (black line) and annealed (red line) Co-doped ITO film. The new spectral features for the annealed sample are indicated with red arrows. The spectra have been normalized at $L_{3}$ edge.

XMCD was detected in TEY. A very weak dichroic effect was, however, observed in the FY XMCD spectra and is indicative of a remanent ferromagnetic ordering (see Figure 6). In the as-deposited film, no remanent XMCD in TEY or FY mode was detected [13] confirming the paramagnetic nature of the $\mathrm{Co}^{2+}$ ions.
Quantitative information regarding the orbital $\left(\mu_{L}\right)$ and spin $\left(\mu_{S}\right)$ components to the total magnetic moment per Co ion $\left(\mu_{\mathrm{TOT}}\right)$ was calculated by applying sum rules $[21,23]$, where we have explicitly included the magnetic dipole term $\left(\mu_{T}\right)$ for the as-deposited and annealed films. For the Co foil the number of holes used for our calculations was 2.49 ([21]), 


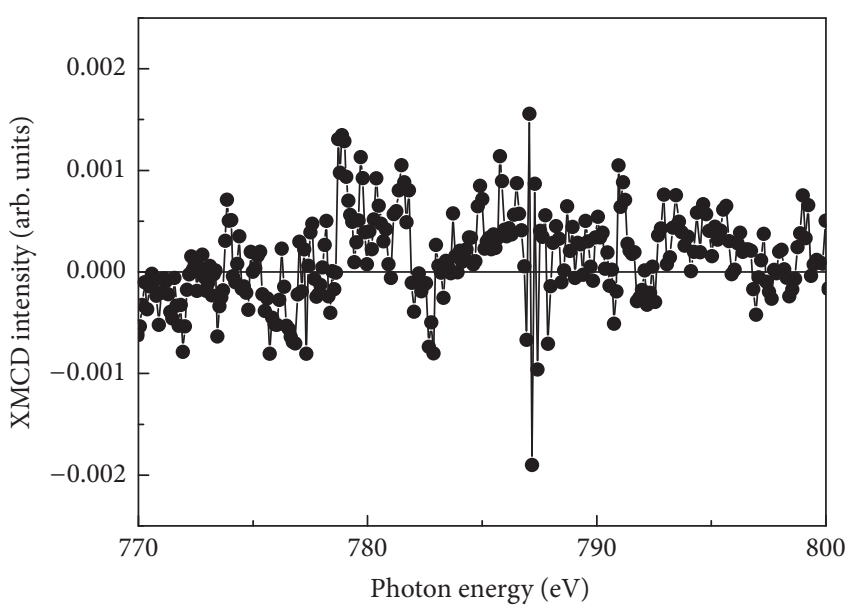

(a)

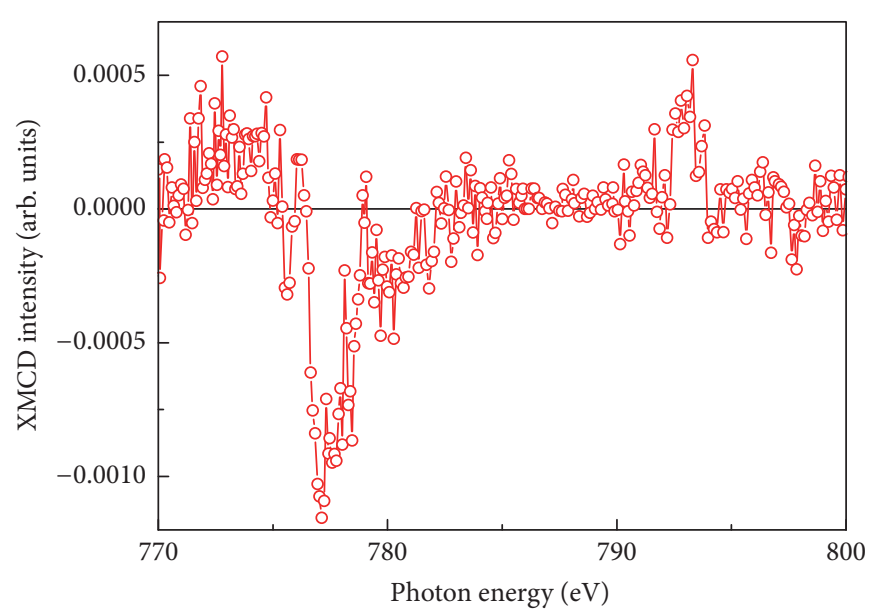

(b)

FIgure 6: (a) TEY and (b) FY XMCD spectra at $2 \mathrm{~K}$ held at remanence for a Co-doped ITO film annealed at $975 \mathrm{~K}$.

TABLE 1: Spin $\left(\mu_{S}\right)$ and orbital $\left(\mu_{L}\right)$ components of the total magnetic moment per Co ion $\left(\mu_{\mathrm{TOT}}\right)$ at $4 \mathrm{~K}$ in a magnetic field of $4 \mathrm{~T}$ calculated using the XMCD sum rules.

\begin{tabular}{lccc}
\hline Sample & $\mu_{S}\left(\mu_{B} / \mathrm{Co}\right)$ & $\mu_{L}\left(\mu_{B} / \mathrm{Co}\right)$ & $\mu_{\mathrm{TOT}}\left(\mu_{B} / \mathrm{Co}\right)$ \\
\hline Co foil & $1.55 \pm 0.11$ & $0.14 \pm 0.04$ & $1.69 \pm 0.12$ \\
As-deposited & $2.17 \pm 0.09$ & $0.56 \pm 0.03$ & $2.73 \pm 0.10$ \\
Annealed & $2.15 \pm 0.12$ & $0.41 \pm 0.04$ & $2.56 \pm 0.13$ \\
\hline
\end{tabular}

but, in the case of the Co-doped samples, the number of holes was set to 3.00. The results of the analysis are shown in Table 1. The value for the Co foil total moment is in excellent agreement with the theoretical value for Co metal $\left(1.72 \mu_{B}\right)$ [24]. Within the error it is evident that the as-deposited and annealed films have a similar $\mu_{S}$, but there is a marked reduction in $\mu_{L}$ for the annealed film. The quenching of $\mu_{L}$ is most likely due to a lowering in the degeneracy of the states arising from a change in the crystal field at the Co sites due to the clustering of the Co dopants. The value of $\mu_{\mathrm{TOT}}$ is lower than the theoretical value for the isolated $\mathrm{Co}^{2+}$ ion $\left(\sim 3.87 \mu_{B}([25])\right)$ with $\mu_{\text {TOT }}$ reduced further upon annealing and closer to that of pure Co metal. Rode et al. observed similar reductions in the sum rule calculated magnetic moments [24]. They suggested that the origins of the reduction may be due to a lack of magnetic saturation. However, this is unlikely to be the case for our study conducted in a field of $4 \mathrm{~T}$. On the other hand, antiferromagnetic interactions between nearest neighbor $\mathrm{Co}^{2+}$ ions could also be responsible for the low value of the moment [24]. The rise in magnetization upon UHV annealing should therefore be largely ascribed to defects introduced during the formation of metallic Co clusters, as evidenced by changes in the XAS and XMCD spectra as well as the blocking temperature in the magnetization versus temperature response. The formation of oxygen vacancies during annealing provides a contribution to the overall ferromagnetic response by significantly enhancing the carrier density. Furthermore, these results demonstrate that the magnetism in the as-deposited Co-doped ITO film cannot be ascribed to cluster formation. We therefore class the ferromagnetic response in as-deposited Co-doped ITO as intrinsic.

\section{Summary and Conclusions}

In summary, we have shown that an increase in magnetization upon UHV annealing of Co-doped ITO at $975 \mathrm{~K}$ is due to the formation of ferromagnetic metallic Co clusters which have a lower magnetic moment per Co site than the Co impurities in the as-deposited film. Multiplet features in the Co $L_{2,3}$ edge XAS spectra measured in both TEY and FY from the as-deposited film indicate that the Co impurities are divalent. This is also the case for the annealed sample at the near surface region, but bulk sensitive FY TEY spectra reveal the presence of substantial Co metal clustering. The results demonstrate that clustering is not the origin of the ferromagnetism observed in the as-deposited Co-doped ITO film. This study should therefore inspire further work to understand the fundamental role of intrinsic lattice defects in dilute magnetic oxides.

\section{Conflicts of Interest}

The authors declare that they have no conflicts of interest regarding the publication of this paper.

\section{Acknowledgments}

This work was funded by the Engineering and Physical Sciences Research Council. The authors would like to thank Richard Mott for his support during the experiments at Diamond Light Source and the Science and Technology Facilities Council for access to the SQUID instrument. 


\section{References}

[1] H. Ohno, "Making nonmagnetic semiconductors ferromagnetic," Science, vol. 281, no. 5379, pp. 951-956, 1998.

[2] S. A. Wolf, D. D. Awschalom, R. A. Buhrman et al., "Spintronics: a spin-based electronics vision for the future," Science, vol. 294, no. 5546, pp. 1488-1495, 2001.

[3] S. X. Zhang, W. Yu, S. B. Ogale et al., "Magnetism and anomalous Hall effect in Co- (La,Sr) $\mathrm{TiO}_{3}$," Physical Review BCondensed Matter and Materials Physics, vol. 76, no. 8, Article ID 085323, 2007.

[4] Y. J. Lee, M. P. de Jong, and R. Jansen, "Magnetism and heterogeneity of Co in anatase $\mathrm{Co}: \mathrm{TiO}_{2}$ magnetic semiconductor," Applied Physics Letters, vol. 96, no. 8, Article ID 082506, 2010.

[5] M. A. Garcia, E. Fernandez Pinel, J. De La Venta et al., "Sources of experimental errors in the observation of nanoscale magnetism," Journal of Applied Physics, vol. 105, no. 1, Article ID 013925, 2009.

[6] M. Venkatesan, C. B. Fitzgerald, and J. M. D. Coey, "Unexpected magnetism in a dielectric oxide," Nature, vol. 430, no. 7000, p. 630, 2004.

[7] N. H. Hong, J. Sakai, N. Poirot, and V. Brizé, "Room-temperature ferromagnetism observed in undoped semiconducting and insulating oxide thin films," Physical Review B, vol. 73, no. 13, Article ID 132404, 2006.

[8] J. Stankiewicz, F. Villuendas, and J. Bartolomé, "Magnetic behavior of sputtered Co-doped indium-tin oxide films," Physical Review B, vol. 75, no. 23, Article ID 235308, 2007.

[9] H. S. Kim, S. H. Ji, H. Kim et al., "Observation of ferromagnetism and anomalous Hall effect in laser-deposited chromiumdoped indium tin oxide films," Solid State Communications, vol. 137, no. 1-2, pp. 41-43, 2006.

[10] J. Philip, N. Theodoropoulou, G. Berera, J. S. Moodera, and B. Satpati, "High-temperature ferromagnetism in manganesedoped indium-tin oxide films," Applied Physics Letters, vol. 85, no. 5, pp. 777-779, 2004.

[11] A. M. H. R. Hakimi, M. G. Blamire, S. M. Heald et al., "Donorband ferromagnetism in cobalt-doped indium oxide," Physical Review B, vol. 84, no. 8, Article ID 085201, 2011.

[12] A. M. H. R. Hakimi, N. Banerjee, A. Aziz, J. W. A. Robinson, and M. G. Blamire, "Estimating the spin diffusion length of semiconducting Indium Tin Oxide using Co/Indium Tin Oxide/Co spin valve junctions," Applied Physics Letters, vol. 96, no. 10, Article ID 102514, 2010.

[13] A. M. H. R. Hakimi, F. Schoofs, R. Bali et al., "Origin of magnetism in cobalt-doped indium tin oxide thin films," Physical Review B, vol. 82, no. 14, Article ID 144429, 2010.

[14] J. M. D. Coey, P. Stamenov, R. D. Gunning, M. Venkatesan, and K. Paul, "Ferromagnetism in defect-ridden oxides and related materials," New Journal of Physics, vol. 12, Article ID 053025, 2010.

[15] R. P. Panguluri, P. Kharel, C. Sudakar et al., "Ferromagnetism and spin-polarized charge carriers in $\mathrm{In}_{2} \mathrm{O}_{3}$ thin films," Physical Review B, vol. 79, no. 16, Article ID 165208, 2009.

[16] V. N. Antonov, L. V. Bekenov, L. P. Germash, and N. A. Plotnikov, "X-ray magnetic dichroism in the cobalt-doped indium tin oxide from first principle calculations," Journal of Solid State Physics, vol. 2013, Article ID 213594, 10 pages, 2013.

[17] H. Baqiah, N. B. Ibrahim, S. A. Halim, M. H. Flaifel, and M. $\mathrm{H}$. Abdi, "The role of cobalt doping on magnetic and optical properties of indium oxide nanostructured thin film prepared by sol-gel method," Materials Research Bulletin, vol. 63, pp. 147$154,2015$.

[18] F. S. Galasso, Structure and Properties of Inorganic Solids, Pergamon Press, Oxford, UK, 1970.

[19] A. M. H. R. Hakimi, Magnetism and spin transport studies on indium tin oxide [Ph.D. thesis], University of Cambridge, Cambridge, UK, 2011.

[20] J. M. D. Coey, M. Venkatesan, and C. B. Fitzgerald, "Donor impurity band exchange in dilute ferromagnetic oxides," Nature Materials, vol. 4, no. 2, pp. 173-179, 2005.

[21] C. T. Chen, Y. U. Idzerda, H.-J. Lin et al., "Experimental confirmation of the X-ray magnetic circular dichroism sum rules for iron and cobalt," Physical Review Letters, vol. 75, no. 1, pp. 152155, 1995.

[22] D. H. Kim, J. S. Yang, Y. S. Kim et al., "Effects of high-temperature postannealing on magnetic properties of Co-doped anatase $\mathrm{TiO}_{2}$ thin films," Physical Review B, vol. 71, no. 1, Article ID 014440, 2005.

[23] B. T. Thole, P. Carra, F. Sette, and G. van der Laan, "X-ray circular dichroism as a probe of orbital magnetization," Physical Review Letters, vol. 68, no. 12, pp. 1943-1946, 1992.

[24] K. Rode, R. Mattana, A. Anane et al., "Magnetism of (Zn,Co)O thin films probed by $\mathrm{x}$-ray absorption spectroscopies," Applied Physics Letters, vol. 92, no. 1, Article ID 012509, 2008.

[25] S. Blundell, Magnetism in Condensed Matter, Oxford University Press, 2001. 

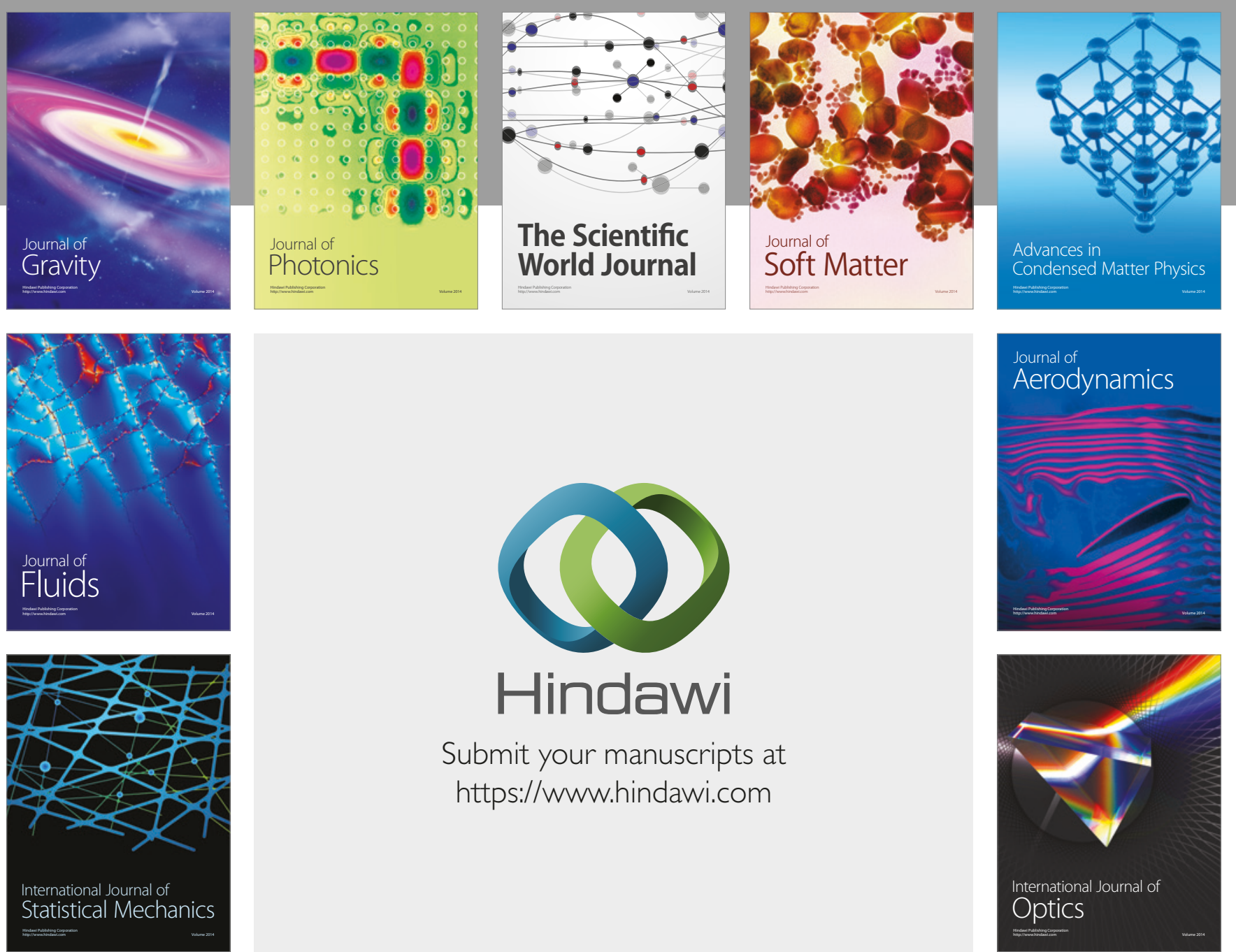

Submit your manuscripts at

https://www.hindawi.com
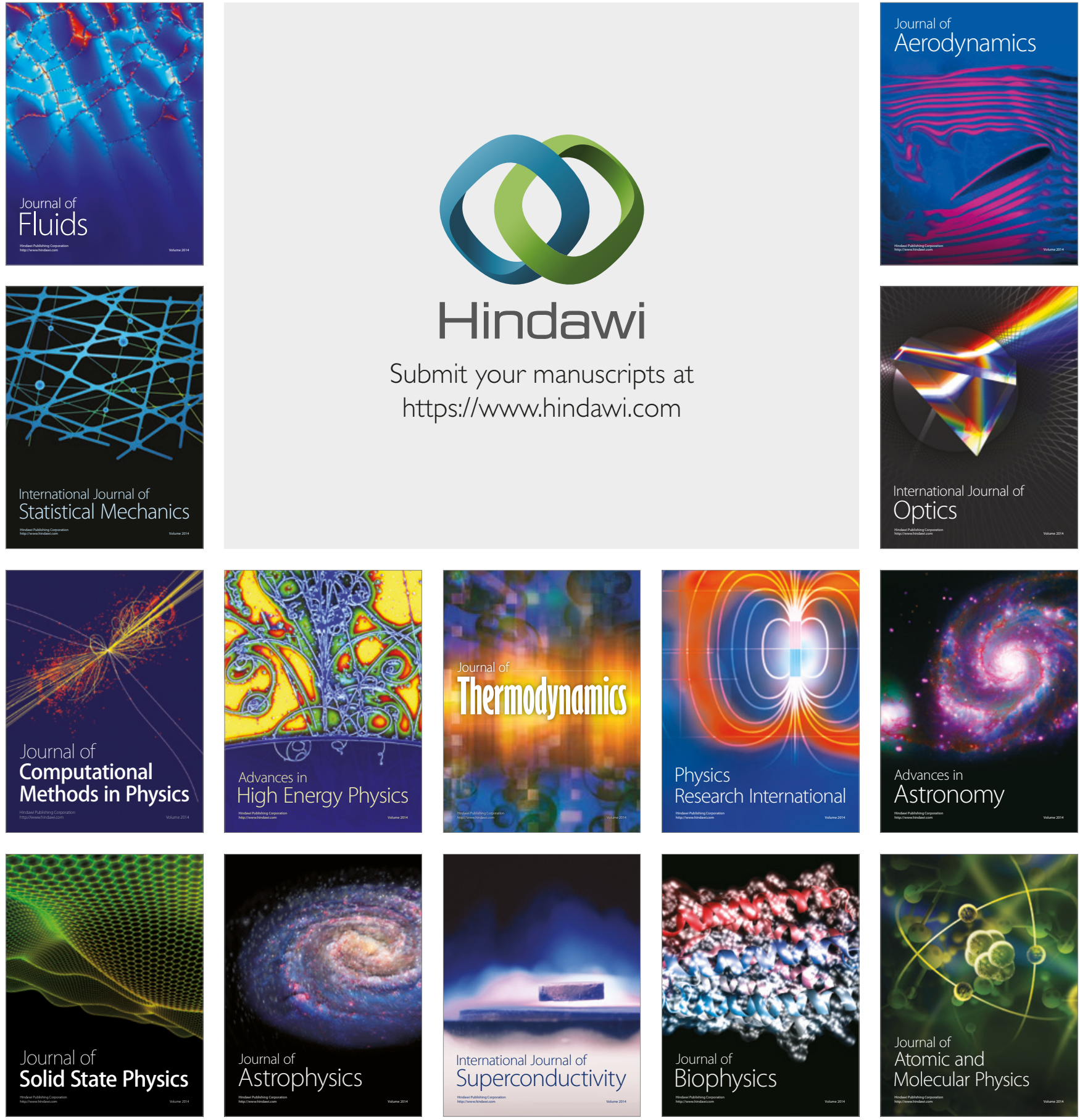